\title{
OP22
}

\section{RETINAL EXPOSURE ASSESSMENT - HORIZONTAL OR VERTICAL ALPHA IRRADIANCE OR ILLUMINANCE? \\ David Sliney}

DOI 10.25039/x46.2019.OP22

from

CIE x046:2019

Proceedings

of the

29th CIE SESSION

Washington D.C., USA, June 14 - 22, 2019

(DOI 10.25039/x46.2019)

The paper has been presented at the 29th CIE Session, Washington D.C., USA, June 14-22, 2019. It has not been peer-reviewed by CIE.

(C) CIE 2019

All rights reserved. Unless otherwise specified, no part of this publication may be reproduced or utilized in any form or by any means, electronic or mechanical, including photocopying and microfilm, without permission in writing from CIE Central Bureau at the address below. Any mention of organizations or products does not imply endorsement by the CIE.

This paper is made available open access for individual use. However, in all other cases all rights are reserved unless explicit permission is sought from and given by the CIE.

CIE Central Bureau

Babenbergerstrasse 9

A-1010 Vienna

Austria

Tel.: +4317143187

e-mail: ciecb@cie.co.at

www.cie.co.at 


\title{
RETINAL EXPOSURE ASSESSMENT - HORIZONTAL OR VERTICAL ALPHA IRRADIANCE OR ILLUMINANCE?
}

\author{
David H. Sliney \\ Department of Environmental Health Science and Engineering \\ Johns Hopkins University School of Public Health, Fallston, MD, USA \\ dsliney1@jhu.edu; dave.sliney@gmail.com
}

DOI $10.25039 / \times 46.2019 .0 P 22$

\begin{abstract}
There have been a number of methods to measure ambient illumination for the purpose of evaluating both visual and non-visual effects in a given environment. With the discovery of the photosensitive retinal ganglion cells, much of the attention has related to the most relevant spectral functions, and this was also the focus of the recently published CIE Standard, S026:2018, "System for Metrology of Optical Radiation for ipRGC-Influenced Responses to Light." However, S-026 also notes that the field-of-view of the subject observer significantly affects the actual retinal exposure. It is therefore important to review the spatial aspects of retinal exposure. Since the head is normally upright, many have suggested measuring vertical (rather than horizontal) illuminance (lux) or field-averaged luminance $\left(\mathrm{cd} \cdot \mathrm{m}^{-2}\right)$. However, perhaps both measures have merit, since the human visual field is actually aimed downward in most settings. Measurements of spectral reflectance within this field can be most revealing.
\end{abstract}

Keywords: Visual Field, Retinal Ganglion Cells, Melanopic, Retina, ipRGCs

\section{Assessing Retinal Exposure}

It is first useful to review the traditional photometric quantities that most relate to retinal light exposure. Vision scientists most generally use the key externally measureable quantity - the luminance $\left(\mathrm{cd} \cdot \mathrm{m}^{-2}\right)$, because retinal illumination is directly related to luminance. However, luminance alone is not the only variable that influences retinal illumination - area of the pupil determines the total luminous power entering the eye. Past measures of actual retinal exposure in vision science have generally employed the unit of the Troland ( $T d)$, which adds the critical factor of pupil size to the information available as luminance, so the retinal illumination in Td is the product of the pupillary area $A_{p}$ in $\mathrm{mm}^{2}$ and the luminance $L_{v}$ in $\mathrm{cd} \cdot \mathrm{m}^{-2}$ (i.e., $\mathrm{Im}^{-2} \cdot \mathrm{m}^{-2} \cdot \mathrm{sr}^{-1}$ ). To some purists the $\mathrm{Td}$ is too much a strange mixture of units and not $\mathrm{SI}$; nonetheless, it cannot be confused when properly applied, and basic research of afterimages and other retinal effects has used this quantity in practice. Even though dimensionally the $\mathrm{Td}$ could be reduced to candelas, which although dimensionally correct, this has only caused confusion when retinal illuminance is expressed in cd. In photobiology and in optical radiation safety, spectrally weighted radiometric quantities are almost always used (Sliney, 2002; Sliney, 1980). Radiance $\mathrm{L}_{\mathrm{e}}$ in $\mathrm{W} \cdot \mathrm{m}^{-2} \cdot \mathrm{sr}^{-1}$ holds sway in photobiological standards of lamp safety as applied in CIE S-009:2002 (CIE,2002). In ophthalmic-instrument safety standards the limits are expressed directly as irradiance at the retina and are typically calculated for a standard pupil size to correspond to applicable, equivalent, lamp-safety radiance limits (with some added considerations). Thus, there are a number of methods to quantify both instantaneous retinal irradiance and time-averaged retinal exposure (Sliney, 2005; 2007).

If spectral weighting functions are applied, much more information is included. The $\alpha-$

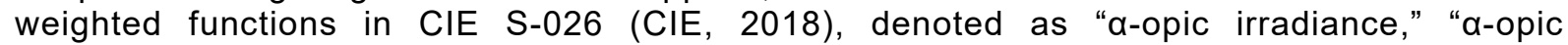
radiance," " $\alpha$-opic radiant flux" are examples. The $\alpha$-opic is generic to relate to one of the five photoreceptors (such as a rod, a cone or a melanopic ipRGC). But in illuminating engineering, in the absence of spectral radiance and luminance measurements, the photometric quantities, of vertical or horizontal illuminance have been used, engendering a debate of which is more relevant to describe effects for health and well being. How relevant is either of these measures? Neither considers the human visual field-of-view (FOV). The human FOV does not extend to the hemisphere of light implied in the definition of illuminance (CIE 17-550). 


\section{The Human Visual Field}

Visual field assessment for diagnostic purposes (e.g., monitoring glaucoma) in clinical settings is termed "perimetry." When performed in ophthalmic clinics (Spector, 1990) only the visually important central $\pm 30^{\circ}$ has been measured traditionally (Figure 1). In vision science the extreme vertical limits in an illuminated indoor setting has been taken as $+45-50^{\circ}$ (or mesopic as great as $+60^{\circ}$ ) and $-70-75^{\circ}$. Although many texts indicate a practical horizontal FOV of $\pm 60^{\circ}$ an individual's most extreme limit of visual detection can exceed $90-100^{\circ}$ to the side (temporal), but of course the FOV of a single eye is normally limited on the nasal (medial) side to no more than $\sim 60^{\circ}$. Although retinal ganglion cells (RGCs) are absent in the visual centre (the fovea and central macula) they cover the entire retina (Esquiva, 2017). The type of visual field measure from a conventional Goldmann perimeter is shown in Figure 1.

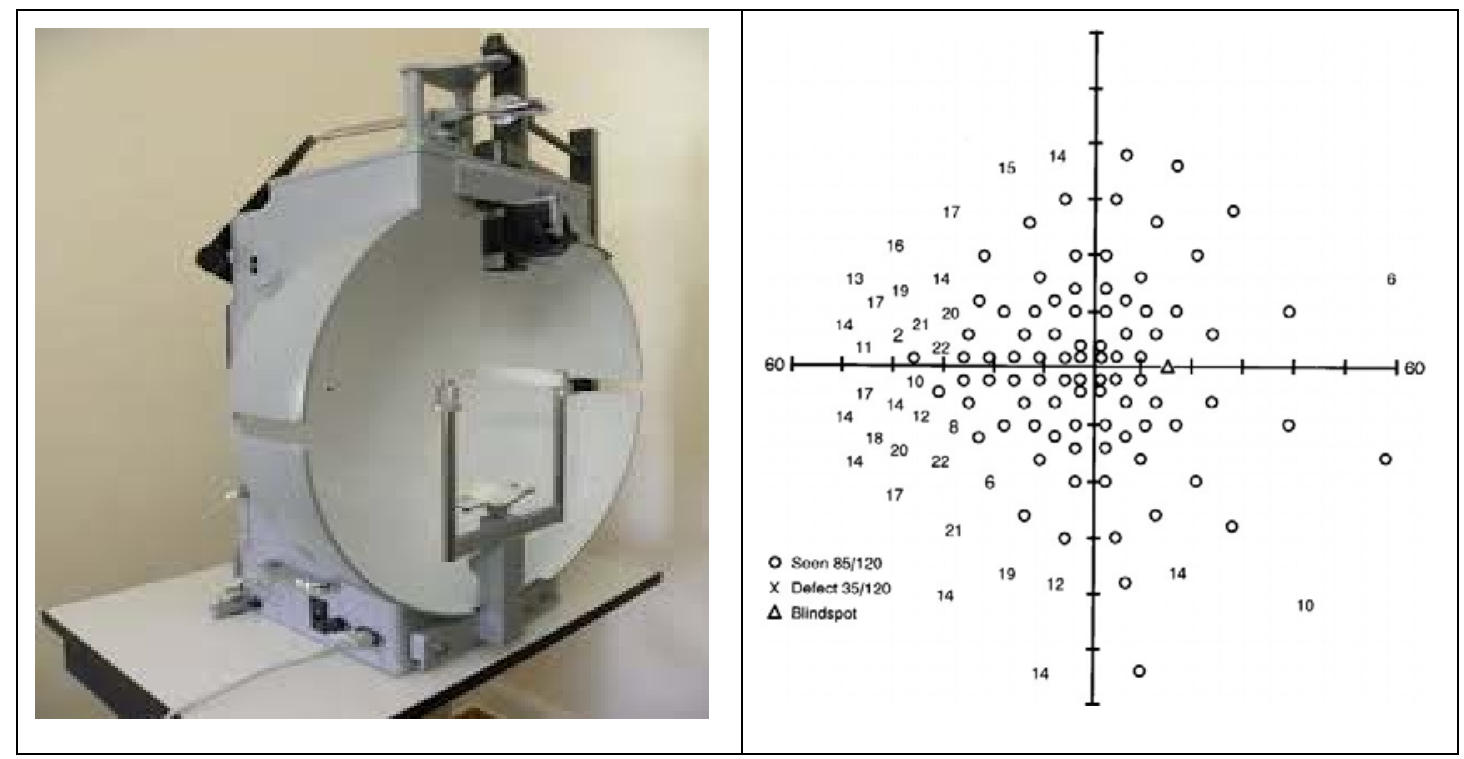

Figure 1 - Perimetric Visual Field. A typical Goldmann Perimeter is shown at left. A printout of a visual field measurement from a similar clinical instrument is shown in the right panel.

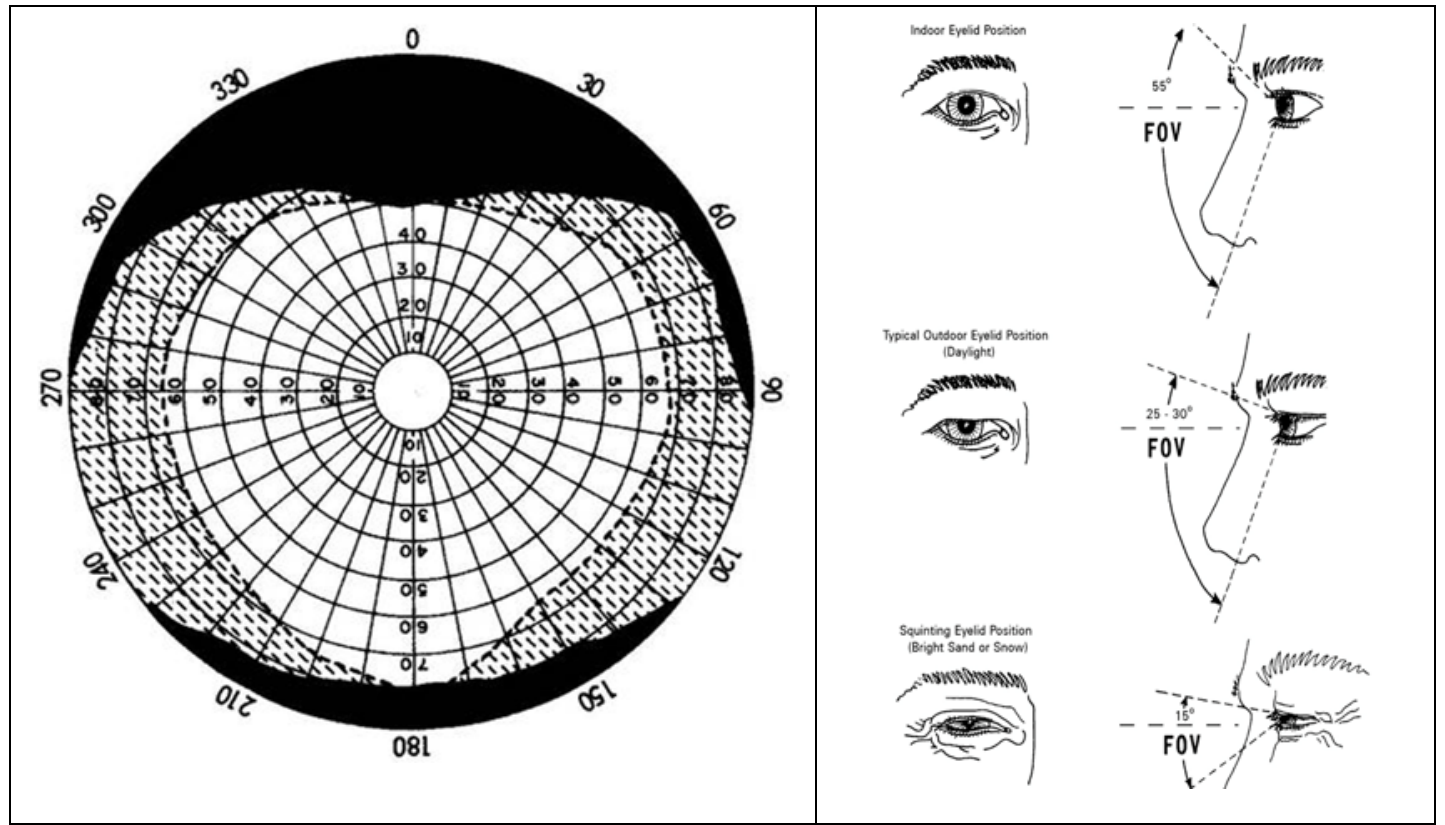

Figure 2 - The Human Visual Field Varies with Scene Luminance. Indoor limits (left) is for indoor photopic vision (NASA); but, upper FOV limit drops with outdoor luminance. 
The varying field-of-view (FOV) - at least in the outdoor environment - as shown in the right panel of Figure 2, cannot be neglected in quantifying the spectral variations and describing retinal exposure. The most important variable is the upper limit of the FOV. As shown in the left panel of Figure 2, the upper limit is frequently taken around $+45-50^{\circ}$ in textbooks and handbooks, and this is factored into metrics to assess the overhead glare from luminaires. However, the upper lid lowers involuntarily (neurobehaviorally) when one walks outside into sunlight (Deaver, 1996). Studies of a sampling of adults viewing a ganzfeld illuminated by sunlight by Deaver et al. (1996) showed a very substantial variance among individuals, but the average value of the angle $\phi_{F} \mathrm{OV}$, which is the vertical angular extent above the horizon varies with the scene luminance $L_{v}$ as:

$$
\phi \phi_{\mathrm{FOV}}=34^{\circ}-0.0013 \mathrm{LV}
$$

where

$\phi_{F O V}$ is the vertical FOV in degrees in outdoor daylight

$L v \quad$ is the luminance of the area of the sky or building being viewed (averaged over $1 \mathrm{sr}$ ).

Although Eqn. (1) is an average value, it should be emphasized that there was a substantial variance in values between individuals, with lid openings differing by two fold for the exact same luminance condition; values readily ranged by $\pm 15^{\circ}$. The mean difference between dark brown irides (higher FOV) and green/blue irides (lower) as two groups was only $\pm 4^{\circ}$. Most persons are unaware of this change in the upper-lid position and a change in field of view when outdoors. I often ask a class at lunchtime to estimate their upper FOV in the classroom before departing (usually just over $45^{\circ}$ ) and then walk out into sunlight and raise their hand above their head to estimate the reduced FOV. Almost everyone is very surprised - and impressed that they never noticed this visual limitation before. At one time we thought that the brow ridge was the limiting factor for the upward field-of-view, but it turned out to be the upper lid, which has a finite thickness and normally rides at the upper margin of the pupil.

\section{Quantifying Retinal Irradiance}

The retinal irradiance $E_{\text {ret }}$ when directly viewing a light source, such as a computer screen can be calculated from a measurement of screen radiance L. Figure 2, below, shows the angular relation between a source-plane dimension $D\left\llcorner\right.$ and the corresponding dimension $d_{r}$ at the retina, where the effective focal length in air of the adult (Gullstrand) eye is taken as $17 \mathrm{~mm}$.

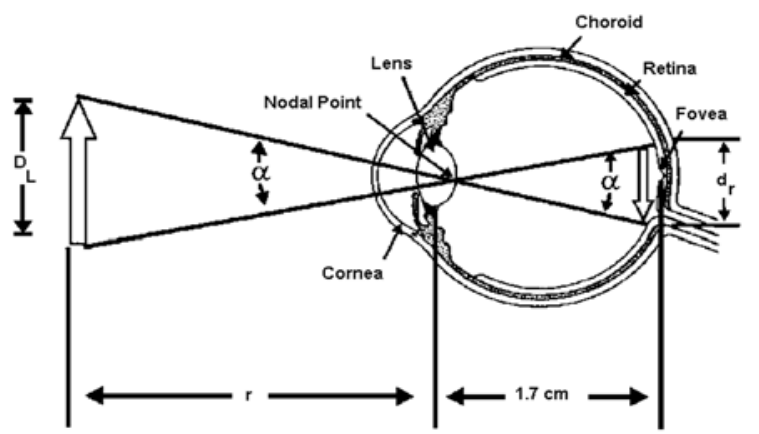

Figure 3 - Viewing a Light Source of Dimension $D_{L}$ at Distance $r$. The diameter of the retinal image is $d_{r}$ varies as linearly as the angle $\alpha$ and the source size $D_{L}$.

From equal angles $\alpha$, and knowledge of the pupil diameter $d_{e}$, it is possible to calculate the retina irradiance $\mathrm{E}_{\text {ret. }}$. The retinal irradiance $\mathrm{E}_{\mathrm{ret}}$ is directly proportional to the source radiance $L$ and to the square of the pupil diameter $d_{e}$. The retinal irradiance $E_{\text {ret }}$ can be therefore calculated as:

$$
E_{\text {ret }}=0.27 \cdot L \cdot \tau \cdot d_{e}^{2}
$$


where $\tau$ (Greek tau) is the transmittance of the ocular media (i.e., the cornea, aqueous, lens and vitreous).

Equation (1) is directly applicable for calculation of retinal irradiance in the central area of the retina - the macula (Sliney, 2005). However, it will over-estimate the exposure at large angles, varying approximately as the cosine of the angle off-axis, since, although the distance from the nodal point to the curved retina decreases with increasing angle, but the irradiance at the pupil plane is decreasing approximately as the cosine of the off-axis angle. Thus, for a 1 -sr $\left(\sim 57^{\circ}\right)$ FOV, Eqn. (1) is a very reasonable estimate of retinal irradiance. The greatest uncertainty relates to the pupil diameter $d_{e}$, which can vary significantly between individuals for the same luminance field. Since the retinal irradiance varies as the square of the pupil diameter - Eqn. (1) - a corresponding change in retinal irradiance can be significant, and two individuals viewing the same sunlit scene outdoors can experience a retinal irradiance - both localized and over-all - with as much as a 2-4 times different (Harley, 2018) in an outdoor setting. Since the ipRGCs play a major role in determining the retinal irradiance for any given viewing condition and the illuminated area of the inferior retina, we should ask whether individuals with a larger pupil size or reduced lid movement have abnormal ipRGC response. There seems to be a fertile area for research. For example, could the sub-population suffering from an increased risk of age-related macular degeneration have experienced a much higher macular irradiance over a lifetime?

It would be useful to conduct measurements of pupil size, vertical field-of-view (FOV) and spectral content were made in several indoor and outdoor settings to demonstrate the appropriateness of vertical or horizontal illuminance/irradiance as surrogates for estimating relative retinal exposures.

\section{Measurements}

\subsection{Measurement Objectives}

Although both horizontal and vertical illuminance/irradiance have been proposed and employed as metrics for circadian and health studies, they can be misleading if either spectral content is ignored or if the typical FOV is ignored. For an individual retinal exposure the pupil size is ignored in these measures and this varies significantly in the same luminance setting. However, the importance of spatial variation in one's environmental light field can clearly be shown to affect the retinal level. Good lighting practice to reduce glare prescribes lower luminance below $\sim 45^{\circ}$; thus, a vertical illuminance or irradiance collects much of the overhead luminaire contribution that does not directly reach the retina. On the other hand a horizontal measure certainly does not adequately measure the light reflected from vertical surfaces. Does this mean that neither measure should be used? In human factors (ergonomic) handbooks, the normal line-of-sight has generally been taken for indoor sitting positions as 15. However, this value was developed from observations of office work prior to the development of computer screens. My observations of individuals in office settings and in airports and rail stations viewing their adjustable computer laptop screens seem to indicated that the slightly downward gaze angle of $-15^{\circ}$ is maintained, with the screen adjusted to that angle for normal view. Observing populations viewing their cell phone almost always indicated a much stronger downward gaze angle of even exceeding - 60o. These simple observations argues for a downward facing measurement of spatially averaged spectral irradiance or spectral radiance. For example, a $1 \mathrm{sr}$ (approximately $\pm 28^{\circ}$ ) hood placed over a cosine detector, whether calibrated for spectral irradiance, for illuminance, for total irradiance, or even for an alpha-weighted spectral response could provide a far better indication of central retinal exposures. As a field trial, a 1-sr or 2-sr hood would provide either spatially averaged radiance or irradiance, and for a 1-sr FOV, the values would be numerically equivalent. For example, if a 1-sr hooded illuminance detector indicated $500 \mathrm{Ix}$, this would also indicated a spatially averaged luminance of $500 \mathrm{~cd} \cdot \mathrm{m}^{-2}$.

\subsection{Spatially Averaged Irradiance/Radiance}

Field measurements of spatially averaged irradiance - both indoors and outdoors - were conducted with detectors fitted with a 1-sr hood. A Gigahertz-Optik Model X11 Optometer, Model RW-3705-4, was employed to conduct the pilot measurements. The two detectors were each fitted with a 1-sr hood; these were a Model RW-3705-4 Radiometric Detector head, 
and a Model UV-3709-4, Blue-Light Hazard Detector head. While neither detector was clearly meant to measure "alpha-irradiance" or "alpha radiance," they indicated a ratio of blue-lightto-total irradiance for demonstration purposes. Although the blue-light-hazard function $B(\lambda)$ has a shorter-wavelength peak than a presumed melanopic function, any blue-indigo-violet band measurement in the short wavelength range (Brainard, 2008; Dacey, 2005) still can demonstrate an indicative ratio of short-wavelength light to total illumination. Reference measurements were taken of several types of luminaires (the "source term") and then downward angles of $-15^{\circ},-30^{\circ}$ and $-60^{\circ}$. Measurements were taken over wood floors, fabric furnishings, desktops and outdoor terrain. In almost all cases the ratio of blue/total was significantly less than from the luminaire or overhead sunlight. A ratio of the full sky (horizontal irradiance) was made for outdoor measurements as well. Some characteristic ratios are provided in Table 1, below.

Table 1 - Blue/Total Ratio for Some Viewed Objects

\begin{tabular}{|l|l|l|}
\hline Object in View & Illumination Source & Blue/total Ratio \\
\hline sidewalk & Midday sunlight & 0,18 \\
\hline Green grass & Midday sunlight & 0,09 \\
\hline Sandy soil & Midday sunlight & 0,19 \\
\hline Office desk & 6500 K CCT LED & 0,09 \\
\hline Carpeted floor & 6500 K CCT LED & 0.10 \\
\hline Wooden floor & 6500 K CCT LED & 0.09 \\
\hline Office desk & Incandescent 60-W & 0.08 \\
\hline Carpeted floor & Incandescent 60-W & 0.08 \\
\hline Wooden floor & Incandescent 60-W & 0.07 \\
\hline White tile floor & 6500 K CCT LED & 0.22 \\
\hline
\end{tabular}

\subsection{The Role of Spectral Reflectance of Viewed Surfaces}

The spectral ratios shown above in Table 1 are only representative - to demonstrate the concept, but it should be remembered that high CCT lamps in overhead luminaires contain much more energy at shorter visible wavelengths than an incandescent lamp, but a large fraction of ground surfaces and indoor surfaces reflect less at shorter wavelengths. Figure 4 illustrates some typical spectral reflectance curves (adapted from Sliney, 1980). The spectral quality of all of one's visual surround clearly alters the retinal spectral exposure.

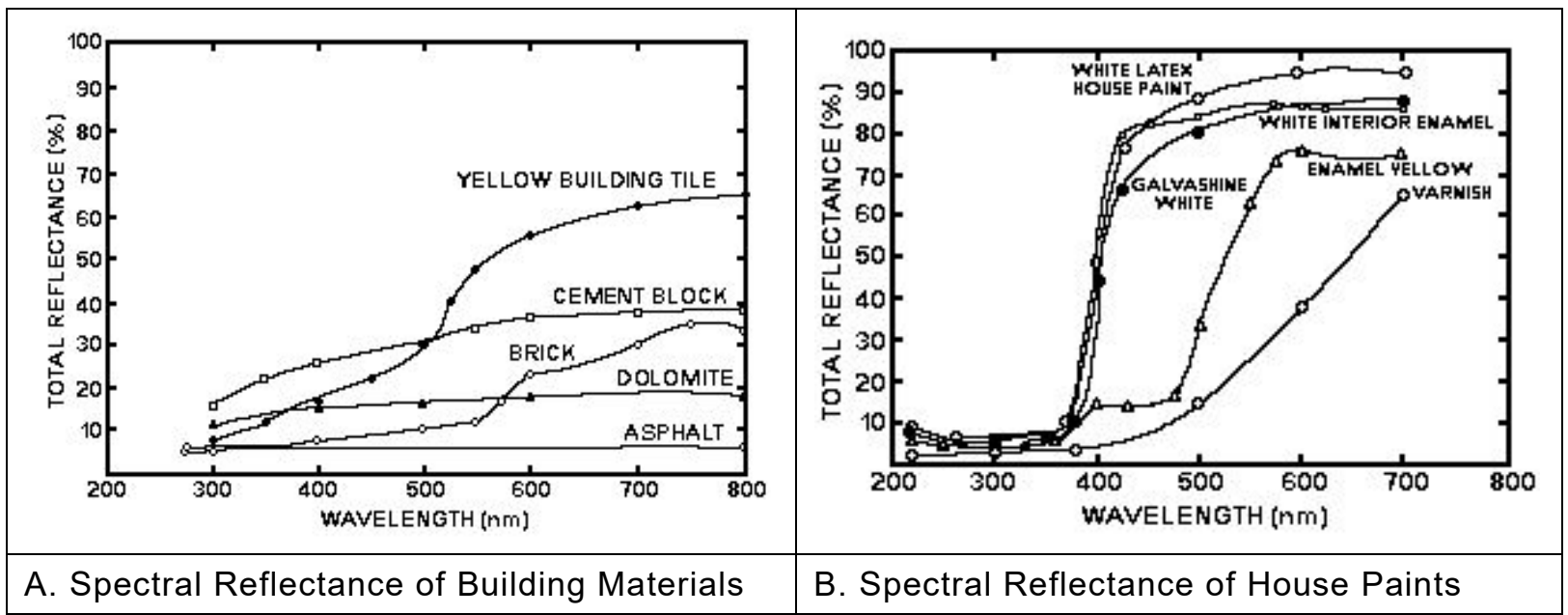

Figure 4 - Spectral Reflectance of Materials within the Field-of-View. Nearly all materials have a lower spectral reflectance at $400-500 \mathrm{~nm}$ than at longer wavelengths. 


\section{Discussion and Conclusions}

The field-of-view (FOV) cannot be neglected in quantifying the spectral variations and retinal irradiance of light stimuli during laboratory and field studies. The use of detectors and wideFOV cameras to monitor viewed scenes should have FOV limits. Employing detectors with a cosine response and hemispherical response can lead to very misleading measures or retinal illumination. Ignoring some of the fundamental findings of visual science and neuroscience can of course also lead to a flawed analysis in studies of "healthy" lighting and non-visual effects of light lighting. It should be remembered that the ipRGCs are far less sensitive to light than the cones (by 1000-fold) and many orders of magnitude less sensitive than the rods; hence, these melanopic receptors have been shown to be well functioning in daylight, but the ipRGCs along with the other photoreceptors also apparently play a very substantial role in providing the brain with indications of light level, time-of-day, transient adaptation, etc. Sadly, it appears that horizontal or vertical illuminance probably tell us very little. If we are really interested in understanding retinal levels across the visible spectrum, we need to measure spatially average spectral radiance.

To this point we have not even considered the fact that the human retinal distribution of certain ipRGCs (e.g., M1 ganglion cells) and their spatial response differ depending upon retinal location, with the inferior retina apparently being more sensitive (Esquiva, 2017; Glickman, 2004).

Irradiance/illuminance meters with a hood to simulate the FOV in that environment and directed along standard directions of gaze provided superior spatial information to assess health and circadian benefits. A simple one-steradian (1-sr) radiance hood is sufficient to cover all of the human central visual field (nearly $60^{\circ}$ linear angle FOV) and because of spatial averaging of radiance, the numerical value of the illuminance or luminance are equal, as well as the numerical value of radiance and irradiance. One can obviously employ a FOV hood to cover and elliptical FOV of $\sim 2-3 \mathrm{sr}$ to include the peripheral retina if that region were shown to be important.

\section{References}

BRAINARD G.C., SLINEY D.H., HANIFIN, J.P., GLICKMAN. G, BYRNE, B, GREESON, J.M., JASSER S, GERNER, E, ROLLAG, M.D. 2008. Sensitivity of the human circadian system to short-wavelength (420-nm) light. J Biol Rhythms. 23(5):379-86.

CIE 2018. CIE S-026:2018. System for Metrology of Optical Radiation for ipRGC-Influenced Responses to Light. Vienna: CIE.

CIE 2002. IEC 62471/CIE S-009:2002. Photobiological Safety of Lamps and Lamp Systems. Vienna: CIE.

DACEY, D.M., LIAO, H.W., PETERSON, B.B., ROBINSON, F.R., SMITH, V.C., POKORNY, J., YAU, K.W., GAMLIN, P.D. 2005. Melanopsin-expressing ganglion cells in primate retina signal colour and irradiance and project to the LGN. Nature, 433: 749-754.

DEAVER, D.M., DAVIS J., SLINEY, D.H. 1996. Vertical visual fields-of-view in outdoor daylight. Lasers and Light, 7(2/3), 121-125.

ESQUIVA, G., LAX, P., PÉREZ-SANTONJ, J.J., GARCÍA-FERNÁNDEZ J.M., CUENCA, N. 2017. Loss of melanopsin-expressing ganglion cell subtypes and dendritic degeneration in the aging human retina. Front. Aging Neurosci., 9(79):1-17.

GLICKMAN G., HANIFIN, J.P., ROLLAG, M.D. WANG, J., COOPER, H., BRAINARD, G. C. 2003. Inferior retinal light exposure Is more effective than superior retinal exposure in suppressing melatonin in humans. J. Biol. Rhythms. 18(1):71-79.

HARLEY, S.K., SLINEY, D.H. 2018. Pupil size in outdoor environments. Health Phys. 115(3):354-359.

SLINEY, D.H. 2005, Exposure geometry and spectral environment determine photobiological effects on the human eye. Photochem Photobiol. 81(3):483-9. 
SLINEY, D.H. 2007. Radiometric quantities and units used in photobiology and photochemistry: recommendations of the Commission Internationale de l'Eclairage (International Commission on Illumination). Photochem. Photobiol. 83:425-432,

SLINEY, D.H. 1980. Safety with Lasers and Other Optical Sources, 1st Ed. New York: Plenum Publishing Corp.

SPECTOR, R.H. 1990. Visual Fields, (in Walker H.K., Hall, W.D., Hurst, J.W., eds.), Clinical Methods: The History, Physical, and Laboratory Examinations. Chapter 116, 3rd Ed. Boston: Butterworths. 